\title{
Successful expression of the Bordetella petrii nitrile hydratase activator P14K and the unnecessary role of Ser115
}

\author{
Weifeng Sun ${ }^{1 \dagger}$, Longbao Zhu ${ }^{2 \dagger}$, Xianggui Chen ${ }^{1}$, Ping Chen ${ }^{1}$, Lingling Yang ${ }^{1}$, Wenwu Ding ${ }^{1}$,
} Zhemin $\mathrm{Zhou}^{3}$ and Yi Liu ${ }^{1 *}$

\begin{abstract}
Background: The activator P14K is necessary for the activation of nitrile hydratase (NHase). However, it is hard to be expressed heterogeneously. Although an N-terminal strep tagged P14K could be successfully expressed from Pseudomonas putida, various strategies for the over-expression of P14K are needed to facilitate further application of NHase.

Results: P14K was successfully expressed through fusing a his tag (his-P14K), and was over-expressed through fusing a gst tag (gst-P14K) at its N-terminus in the NHase of Bordetella petrii DSM 12804. The stability of gst-P14K was demonstrated to be higher than that of the his-P14K. In addition, the Ser115 in the characteristic motif CXLC-Ser115-C of the active center of NHase was found to be unnecessary for NHase maturation.

Conclusions: Our results are not only useful for the NHase activator expression and the understanding of the role of Ser115 during NHase activation, but also helpful for other proteins with difficulty in heterologous expression.
\end{abstract}

Keywords: NHase, Bordetella petrii, P14K, Expression, Ser115

\section{Background}

Nitrile hydratase (NHase) is a multi-subunits industrial enzyme, which can efficiently catalyze the nitrile to the corresponding amide. According to the different metal ions in the active center, the NHase could be classified into the two types: the iron type NHase (Fe-NHase) [1] and the cobalt type NHase (Co-NHase) [2]. Due to the high-value product and high catalytic activity, NHase has attracted wide attention in the chemical industry [3].

NHase is composed of $\alpha$ - and $\beta$-subunits [2]. The molecular masses of the $\alpha$-subunit and $\beta$-subunit are generally less than $30 \mathrm{kDa}$, such as those in Bordetella petrii DSM 12804 ( $\alpha$-subunit, $23.2 \mathrm{kDa} ; \beta$-subunit, $24.1 \mathrm{kDa}$ ) and those in Bacillus pallidus RAPc8 ( $\alpha$-subunit, $24.6 \mathrm{kDa} ; \beta$-subunit, $26.5 \mathrm{kDa})[4,5]$. However, an activator is necessary for the functional expression of NHase, such as those in the Co-NHases from

\footnotetext{
* Correspondence: puter_scholar@163.com

${ }^{\dagger}$ Equal contributors

${ }^{1}$ Key Laboratory of Food and Biotechnology, School of Food and

Biotechnology, Xihua University, Chengdu 610039, China

Full list of author information is available at the end of the article
}

Rhodococcus rhodochrous J1 [6-8], Rhodococcus jostii RHA1 [9] and Pseudomonas putida NRRL-18668 [10] as well as in the Fe-NHases from Pseudomonas chlororaphis B23 [11] and Rhodococcus sp. N-774 [12]. However, certain activators are poorly expressed (barely detectable in SDS-PAGE), such as the activator P14K in the $B$. pallidus RAPc8, Comamonas testosteroni 5-MGAM-4D and P. putida NRRL-18668 [4, 10, 13]. Until recently, the P14K in $P$. putida was successfully expressed through fusing a strep tag at its $\mathrm{N}$-terminal, then a novel NHase maturation mechanism, self-subunit swapping, was discovered [5]. However, the stability of the P14K is not yet favorable for its over-expression. Thus, to further facilitate the application of NHase, other fusion protein tags that are helpful for expression of more stable P14K are required.

Additionally, all the $\alpha$-subunits of NHases share a conserved characteristic motif CXLC-Ser-C in the active center $[1,14,15]$, and the Ser in this motif is proposed to be involved in NHase maturation (Fig. 1) [16-18]. 

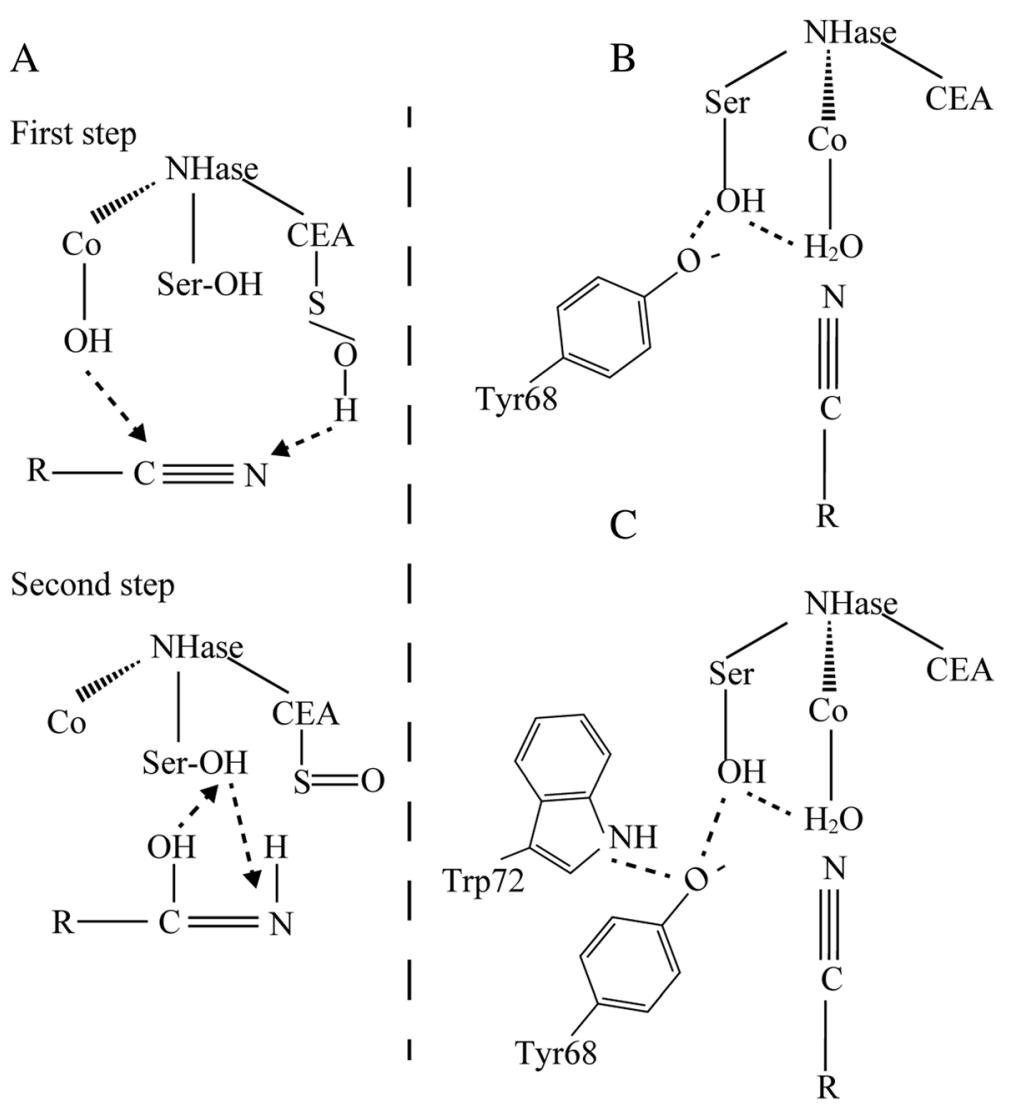

Fig. 1 The catalytic pathway involving Ser1 15 during NHase maturation. a First step: atoms of $\mathrm{O}(\mathrm{CEA})-\mathrm{H}\left(\mathrm{H}_{2} \mathrm{O}\right)$ and $\mathrm{OH}\left(\mathrm{H}_{2} \mathrm{O}\right)-\mathrm{Co}^{3+}$ participates in NHase activation (dashed line). Second step: the proton of $\mathrm{OH}(\mathrm{Ser} 115)$ participates in the proton transfer pathway (dashed line). $\mathbf{b}$ The oxygen of the phenolate of the conserved Tyr68 was ionized by the general base Ser115 during NHase activation. c The oxygen of the phenolate of the conserved Tyr68 was ionized by the general base Ser115 with the help of Tyr72 during NHase activation

However, the necessity of Ser was not studied by the energetic calculation and site-specific mutagenesis.

In this study, the NHase from B. petrii DSM 12804 was researched. The activator P14K was successfully expressed through fusing a his tag, and its expression and stability were further enhanced by fusing a gst tag at its $\mathrm{N}$-terminus. In addition, we found that the Ser115 in the characteristic motif CXLC-Ser-C of the active center of NHase from B. petrii is not necessary for the NHase maturation. These results are useful for the expression of NHase activator and the understanding of the role of Ser115 during the NHase maturation. Further, the strategy for enhancing the stability of P14K is very beneficial for other proteins with difficulty in expression.

\section{Results and discussion}

\section{Expression and purification of NHase}

The full-length genes of NHase $(A B P)$ were obtained from $B$. petrii DSM 12804, in which $A$ gene encodes $\alpha$ subunit, $B$ gene encodes $\beta$-subunit and $P$ gene encodes P14K (Fig. 2). The recombinant expression vector pET$24 \mathrm{a}(+)$ harboring the $A B P$ genes (pET-ABP) was

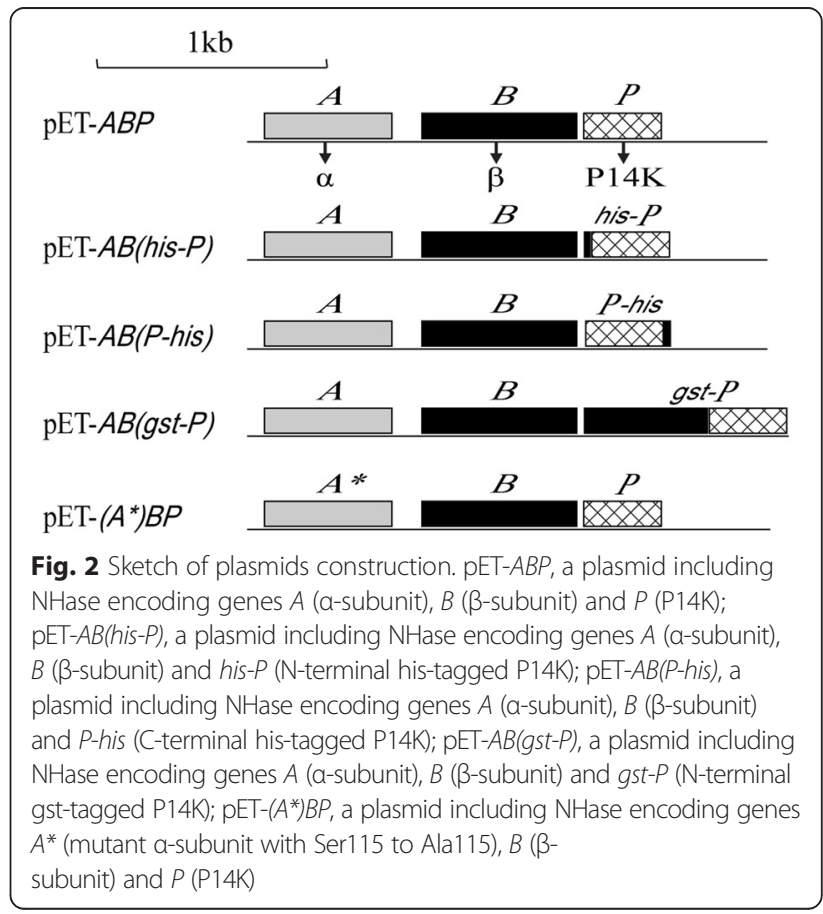


constructed and transformed into E. coli for NHase expression. The calculated molecular mass of recombinant NHase was in accordance with that of the bands by SDS-PAGE analysis (Fig. 3a lane 3). The NHase was successfully purified (Fig. 3a lane 4) and the specific activity was $438.5 \mathrm{U} / \mathrm{mg}$. The molecular mass of NHase was nearly $106 \mathrm{kDa}$ detected by gel filtration chromatography, indicating that the NHase is a tetramer (Fig. 3b).

According to our previous work $[3,5,7,8,19-21]$ and others' reports $[4,13,22]$ about the NHase activators, the $\mathrm{P} 14 \mathrm{~K}$ is necessary for the functional expression of NHase in B. pallidus RAPc8, C. testosteroni 5-MGAM4D and $P$. putida NRRL-18668. Since the amino acid

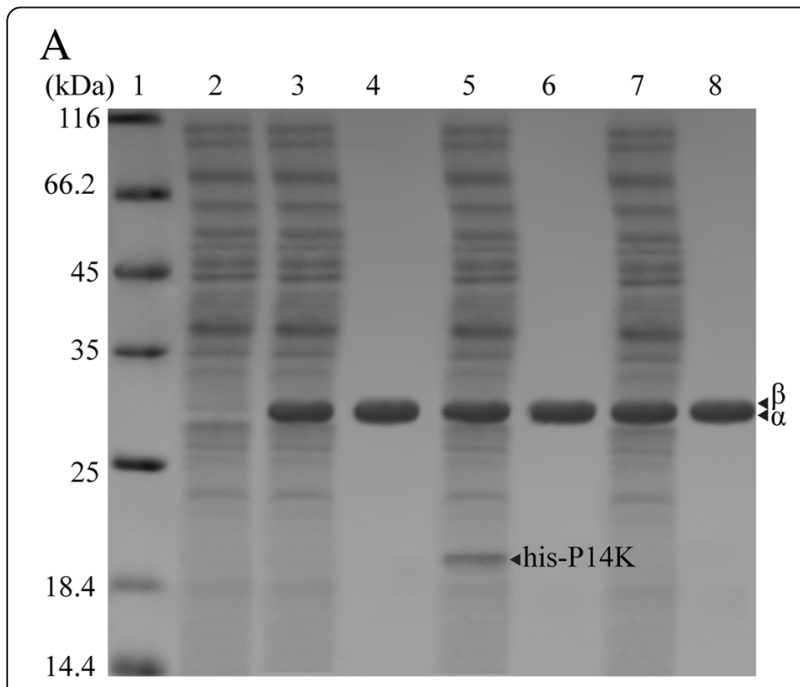

B

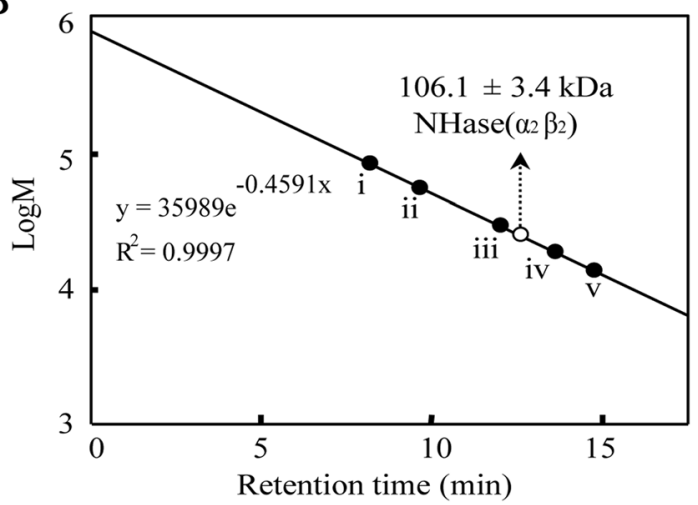

Fig. 3 Expression and purification of recombinant NHases. a 1, mark; 2, control; 3, cell extracts (pET-ABP); 4, purified NHase (pET-ABP); , cell extracts (pET-AB(his-P)); 6, purified NHase (pET-AB(his-P)); 7, cell extracts (pET-AB(P-his)); 8, purified NHase (pET-AB(P-his)). b Molecular mass determination on a Superdex 200 prep grade column. Standard proteins are as follows: (i) thyroglobulin (bovine thyroid) (669 kDa); (ii) ferritin (horse spleen) $(440 \mathrm{kDa})$; (iii) aldolase (rabbit muscle) (158 kDa); (iv) conalbumin (chicken egg white) (75 kDa); and (v) ovalbumin (hen egg) (43 kDa). The open circle represents the determined molecular mass. The values represent the means \pm SD for at least three independent experiments [5] sequence of NHase in B. petrii possessed a high similarity (more than $90 \%$ ) with that in P. putida NRRL18668 , it is proposed that the $\mathrm{P} 14 \mathrm{~K}$ in $\mathrm{B}$. petrii is also necessary for NHase activation. Here, the NHase expressed by $\mathrm{pET}-A B P$ plasmid exhibited enough activity (more than $10 \mathrm{U} / \mathrm{mg}$ ), indicating the successful expression of NHase and P14K, though the P14K band was not detected in SDS-PAGE (Fig. 3a lane 3).

\section{Successful expression of the activator P14K}

The $\mathrm{N}$-terminal residues of a protein are linked to its stability, which is conserved in bacteria $[23,24]$. Nterminal lysine endows a protein short half-life, whereas glycine endows the protein long half-life ( $\mathrm{N}$-end rule) [25]. P14K, hardly detectable in SDS-PAGE, was successfully expressed in the NHase of $P$. putida through molecular modification in the light of $\mathrm{N}$-end rule [21]. To investigate this phenomenon in NHase activator P14K from B. petrii, a his-P14K was designed, in which a his tag was attached to the N-terminus of P14K. Since the N-terminal amino acids of P14K (KDE sequence) were changed to the GSS sequence of the designed his$\mathrm{P} 14 \mathrm{~K}$, the his-P14K would be more stable in terms of the N-end rule. The plasmid pET-AB(his-P) including NHase encoding genes $A$ ( $\alpha$-subunit), $B$ ( $\beta$-subunit) and his-P (his-tagged $\mathrm{P} 14 \mathrm{~K}$ ) was transformed into $E$. coli and was applied for NHase expression (Fig. 2). As a result, the his-P14K was successfully expressed (detectable in SDS-PAGE) (Fig. 3a lane 5) and the enzymatic activity detected in the cell-free extracts was at the same level with that of the original NHase $(120.5 \mathrm{U} / \mathrm{mg})$. Although NHase was successfully purified (Fig. 3a lane 6) and the specific activity was $430.1 \mathrm{U} / \mathrm{mg}$, the amount of P14K expression was still low (Fig. 3a lane 5).

To investigate the influence of the his tag on the Cterminal of P14K, a P14K-his, adding a his tag at the Cterminal of $\mathrm{P} 14 \mathrm{~K}$, was designed. The transformed cells carrying the pET- $A B(P$-his $)$, a plasmid including NHase encoding genes $A$ ( $\alpha$-subunit), $B$ ( $\beta$-subunit) and $P$-his (C-terminal his-tagged P14K), were applied for NHase expression. As a result, the enzymatic activity detected was comparable to that of the original NHase, however, P14K-his was barely detected in SDS-PAGE (Fig. 3a lane 7). These results indicated that the C-terminal of P14K does not play a key role in the expression of P14K.

\section{High expression of P14K attached with a gst tag}

The gst tag $(26 \mathrm{kDa})$ encodes a highly soluble protein, which can help other proteins rapidly fold into a stable protein [26]. To produce a large amount of P14K, a gst$\mathrm{P} 14 \mathrm{~K}$, adding a gst tag at the $\mathrm{N}$-terminus of $\mathrm{P} 14 \mathrm{~K}$, was designed. The transformed cells carrying pET- $A B(g s t-P)$, a plasmid including NHase encoding genes $A$ ( $\alpha$-subunit), $B$ ( $\beta$-subunit) and gst-P (gst-tagged P14K), were 
applied for NHase expression. As shown in Fig. 4 lane 3, a large amount of gst-P14K was detected in SDS-PAGE. The enzymatic activity detected in cell-free extracts was at the same level with that of the original NHase. The recombinant NHase was then successfully purified (Fig. 4 lane 4), and the specific activity was $425.2 \mathrm{U} / \mathrm{mg}$. Together with our previous work that the flexibility of P14K from $P$. putida plays an important role in NHase maturation [3] and the analysis that the molecular weight mass of gst $(26 \mathrm{kDa})$ is nearly two times larger than that of P14K (14 kDa), it is suggested that the P14K possesses a high flexibility indeed.

Moreover, the protein stability of his- $\mathrm{P} 14 \mathrm{~K}$ and gstP14K in the cell-free extracts was compared during storage at room temperature by SDS-PAGE analysis. As shown in Fig. 4 lane 5 and lane 6, the his-P14K band nearly disappeared after 2 days whereas the gst-P14K band decayed to $60 \%$ after 6 days. The gst-P14K was far more stable than his-P14K, indicating that the thermal stability might be an important factor in the P14K expression.

A characteristic motif CXLC-Ser-C has been discovered in all the $\alpha$-subunits of NHases up to now $[1,14,15]$. The Ser between the two Cys in this motif is proposed to be involved in the NHase maturation [16-18], by which the proton is transferred to $-\mathrm{NH}_{2}$ (Fig. 1) [17]. These speculations indicated that the Ser was necessary for the NHase maturation. To investigate the necessity of the Ser, the Ser115 in NHase from B. petrii was researched. An energetic difference of the proton transfer via Ser115 (Fig. 5a type I) and the protein transfer not via Ser115 (direct transfer) (Fig. 5a type II) was compared through quantum chemical calculations using TRITON. As shown in Fig. 5b

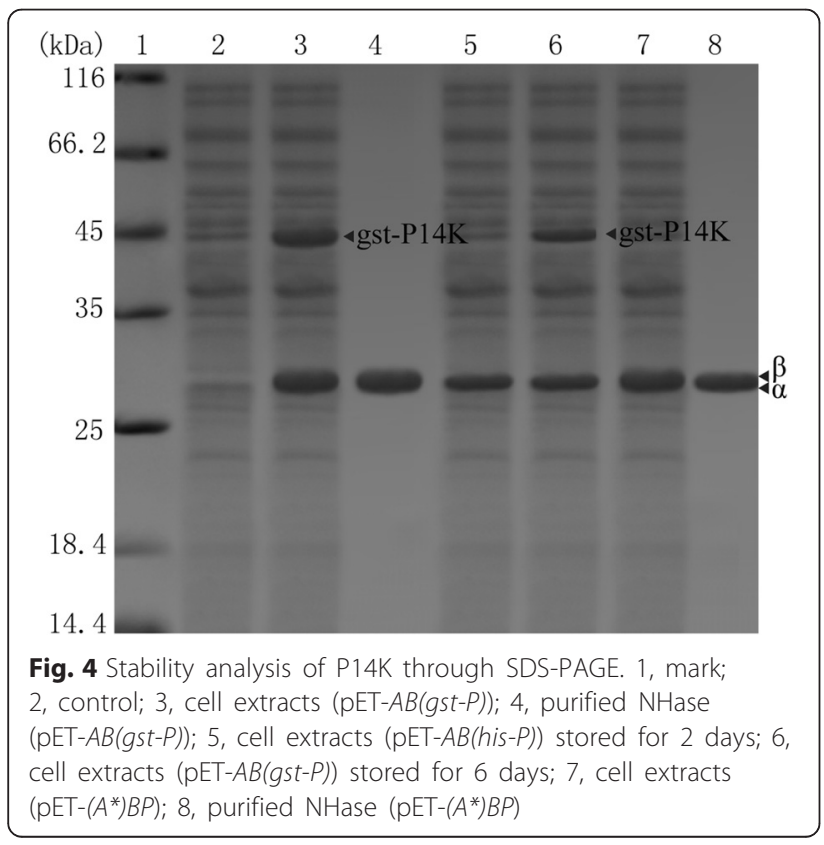

and $\mathrm{c}$, the energy of the proton transferred to $-\mathrm{NH}_{2}$ via Ser115 (type I) $(-13.2 \mathrm{kcal} / \mathrm{mol})$ was more than that of the direct transfer (type II) $(-42.5 \mathrm{kcal} / \mathrm{mol})$, which indicated the type II pathway without the involvement of the Ser115 seemed easier to take place. Thus, a mutant NHase with the Ser 115 mutated to Ala115 was designed to investigate the necessity of the Ser115, considering the structural similarity of the serine and alanine except for the $-\mathrm{OH}$. The transformed cells carrying pET- $\left(A^{*}\right) B P$, a plasmid including NHase encoding genes $A^{*}$ (mutant $\alpha$-subunit with Ser115 to Ala115), $B$ ( $\beta$-subunit) and $P$ (P14K), were applied for the mutant NHase expression (Fig. 4 lane 7). The enzymatic activity $(45.3 \mathrm{U} / \mathrm{mg})$ detected in the cell-free extracts was nearly $40 \%$ of that of original NHase. The NHase was successfully purified (Fig. 4 lane 8), and the specific activity was $170.6 \mathrm{U} / \mathrm{mg}$, also nearly $40 \%$ of that of the purified original NHase. These findings suggested that the Ser in the characteristic motif CXLC-Ser-C of the active center of NHase is not necessary for the catalysis of NHase, however, it could kinetically facilitate the catalysis.

\section{Conclusions}

The activator of NHase from B. petrii DSM 12804 was successfully over-expressed and the Ser115 was verified to be unnecessary for the catalysis of NHase by energetic calculation and site-specific mutagenesis. The strategy for the over-expression of P14K adopted in the paper might be beneficial for other proteins with difficulty in expression.

\section{Methods}

\section{Bacterial strain and plasmids}

NHase genes $(A B P)$ were isolated from $B$. petrii DSM 12804 (accession number for genome, AM902716.1; $\alpha$ subunit, CAP41751.1; $\beta$-subunit, CAP41750.1; P14K, CAP41749.1). The plasmid pET-24a $(+)$ carrying the respective genes $A B P, A B($ his- $P$ ), $A B(P$-his), $A B($ gst-P) and $(A *) B P$ was transformed into E. coli BL21 (DE3) cells (host strain) for heterologous expression.

\section{Plasmids construction}

The NHase genes $A B P$ of $B$. petrii were cloned through the polymerase chain reaction (PCR) with the primers A-up (NdeI recognition sites) and P-down (EcoRI recognition sites) (Table 1). The PCR reaction was conducted using the DNA polymerase KOD-Plus (TOYOBO) under the condition: a pre-denaturation at $94{ }^{\circ} \mathrm{C}$ for $5 \mathrm{~min}$, 30 cycles of denaturation at $98{ }^{\circ} \mathrm{C}$ for $10 \mathrm{~s}$, annealing at $48{ }^{\circ} \mathrm{C}$ for $30 \mathrm{~s}$ and extension at $72{ }^{\circ} \mathrm{C}$ for $90 \mathrm{~s}$, and final extension at $72{ }^{\circ} \mathrm{C}$ for $10 \mathrm{~min}$. The PCR product was analyzed from $1.5 \%(\mathrm{w} / \mathrm{v})$ agarose gel [27]. Afterwards, the cloned NHase genes $A B P$ and the vector pET-24a(+) were treated by the NdeI and EcoRI, and the resultant $A B P$ was then inserted into the pET-24a $(+)$ for the pET- 

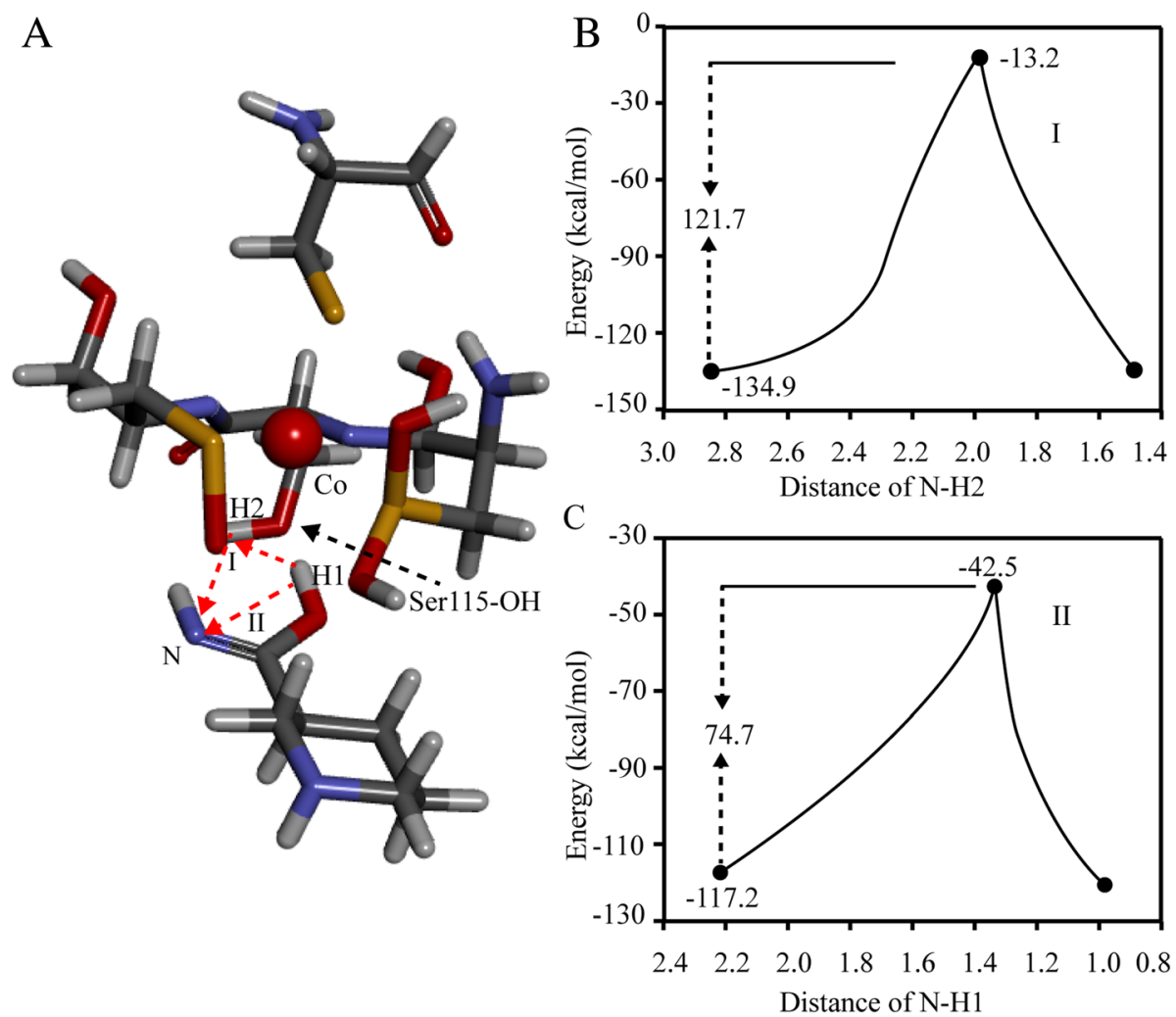

Fig. 5 Energetic calculation of the proposed proton transfer pathway. a The structure of the proposed NHase catalytic model in the active center. The big red ball represents the cobalt ion. The Ser115 was shown by black dashed arrow. The H1 represents the proton of substrate transition state. The $\mathrm{H} 2$ represents the proton of Ser115(OH). The type I is the proton transfer pathway: $\mathrm{H} 1$ (substrate) $\rightarrow \mathrm{H} 2$ (Ser115 of NHase) $\rightarrow \mathrm{N}($ substrate) The type II (direct transfer) is the proton transfer pathway: $\mathrm{H} 1$ (substrate) $\rightarrow \mathrm{N}$ (substrate). $\mathbf{b}$ Energy calculation of proposed transfer pathway (type I): the proton transfer pathway via the proton of $\mathrm{OH}(\mathrm{Ser} 115)$ (red dashed line). $\mathbf{c}$ Energy calculation of proposed transfer pathway (type II): the proton direct transfer pathway (not via the proton of OH(Ser115)) (red dashed line)

$A B P$ construction. The pET-AB(P-his) construction was similar to pET- $A B P$ with the primer pairs A-up and Pdown(his) (EcoRI recognition sites). An overlap extension PCR was conducted to produce plasmid pET$A B$ (his- $P$ ) through two rounds of PCR. The first round of PCR was performed using the primer pairs A-up and B-down(his) and P-up(his) and P-down, respectively, the plasmid pET- $A B P$ was used as the template. The second round of PCR was performed to produce the full-length $A B$ (his- $P$ ), using the primers $\mathrm{A}$-up and $\mathrm{P}$-down and mixing equal molar amounts of the first-round products as template. The $\mathrm{pET}-A B($ gst- $P$ ) construction was similar to pET- $A B$ (his- $P$ ) with the primer pairs A-up and Bdown(gst), and P-up(gst) and P-down (the gst-P gene

Table 1 Oligonucleotide primers used in this study

\begin{tabular}{|c|c|}
\hline Name & Sequence \\
\hline A-up & 5'-GGAATTCCATATGGGGCAATCACACACAC-3' \\
\hline P-down & 5'-CCGGAATTCTCAAGCCATTGCGGCAACGA-3' \\
\hline P-down(his) & 5'-CCGGAATTCTCAGTGATGATGATGATGATGAGCCATTGCGGCAACGA-3' \\
\hline B-down(his) & 5'-TGGCTGCTGCCCATATCTATATCTCCTITCACGCTGGCTCCAGGTAGTCATC-3' \\
\hline P-up(his) & 5'-ATATGGGCAGCAGCCATCATCATCATCATCACAAAGACGAACGGTTTCCATT-3' \\
\hline B-down(gst) & 5'-CTATATCTCCTITCACGCTGGCTCCAGGTAGTCATC-3' \\
\hline P-up(gst) & 5'-GAAAGGAGATATAGATATGTCCCCTATACTAGGTTA-3' \\
\hline$A^{*}$-up & 5'-TCTTCGTCTGCACCCTGTGCGCGTGCTACCCATGGCC-3' \\
\hline$A^{*}$-down & 5'-TGACGGCCATGGGTAGCACGCGCACAGGGTGCAGAC-3' \\
\hline
\end{tabular}

The underlined code represents the gene start code or the Ser115 to Ala115 mutation. Italicized letters denote the Ndel and EcoRI recognition sites, respectively 
synthesized by Sangon Biotech Ltd.). The plasmid pET$(A *) B P$ construction was conducted with the primers $\mathrm{A}^{*}$ up and $\mathrm{A}^{*}$-down using pET-ABP as the template, followed by DpnI digestion to degrade the template plasmid and then transformed into E. coli BL21 (DE3).

\section{NHase expression, purification and activity assay}

The recombinant $E$. coli cells carried the corresponding recombinant plasmids were cultivated in the $\mathrm{TB}$ medium to 0.8 of A600 with kanamycin $\left(37^{\circ} \mathrm{C}\right)$, and then $0.4 \mathrm{mM}$ isopropyl $\beta$-D-thiogalactopyranoside (IPTG) was added. The transformed cells were then cultivated at $24{ }^{\circ} \mathrm{C}$ for $16 \mathrm{~h}$.

The purifier AKTA instrument (GE Healthcare UK Ltd.) was used for proteins purification. All the purifications were kept in the potassium phosphate buffer (KPB, $10 \mathrm{mM}, \mathrm{pH} 7.5)$ at $4{ }^{\circ} \mathrm{C}$. The cell extracts were centrifuged at $18000 \times g$ for $10 \mathrm{~min}$. Protein was preliminarily purified by the ammonium sulfate fractionation (30$70 \%)$ followed by dialysis against KPB. The dialyzed protein ingredients of NHase were purified using a DEAE-Sephacel column $(3 \times 5 \mathrm{~mL})$ (GE Healthcare UK Ltd.) equilibrated with KPB. The fraction containing target proteins was gradually eluted off by a linear concentration of $\mathrm{KCl}(0-0.5 \mathrm{M})$ during purification and then the collected proteins were pooled and followed by ammonium sulfate fractionation (70 \%). After centrifugation, the dissolved precipitate (KPB containing $0.2 \mathrm{M}$ $\mathrm{KCl}$ ) was applied to a HiLoad 16/60 Superdex 200 prep grade column (GE Healthcare UK Ltd.) equilibrated with $0.2 \mathrm{M} \mathrm{KCl}$-containing $\mathrm{KPB}$. The enzymes were then eluted from the HiLoad 16/60 Superdex 200 prep grade column. The fractions containing the enzymes during the purification steps were revealed by SDS-PAGE.

The NHase activity was measured in a reaction system $(500 \mathrm{~L})$ consisted of NHase $(0.1 \mathrm{~g})$, the substrate (20 mM 3-cyanopyridine) and the buffer (10 mM KPB, $\mathrm{pH}$ 7.5), which was placed for $20 \mathrm{~min}$ at $20{ }^{\circ} \mathrm{C}$ and stopped by addition of $500 \mathrm{~L}$ acetonitrile. The product concentration (nicotinamide) was detected with HPLC (high-pressure liquid chromatography) to measure the NHase activity [6]. The amount of protein that was capable of formation of 1 mol nicotinamide in NHase catalysis was defined as one unit of activity.

\section{Homology modeling}

The Modeller 9.7 [28] and the PROCHECK software (http://services.mbi.ucla.edu/SAVES/) were applied for the prediction of protein structure and stereochemical analysis. The final models that displayed good geometry (with less than $1 \%$ of residues in the disallowed region) were used in this study.

\section{Transition state calculation}

TRITON was used for modeling enzymatic reaction, which is freely supported by the National Center of Biomolecular Research (http://ncbr.chemi.muni.cz/triton/) consisting of three modules of MOPAC, MODELLER and DRIVER [29]. The optimal structure and the energy could be obtained and compared by activation energy calculation if the reaction pathway is reasonable. Notably, any improper structures were impossibly got when a proposed reaction pathway was difficult to perform [17].

\section{Abbreviations \\ $\left(A^{*}\right) B P$ : the NHase encoding genes including $A^{*}$ (mutant a-subunit with Ser115 to Ala115), $B$ ( $\beta$-subunit) and $P$ (P14K); $A B P$ : the full-length genes of NHase; Co-NHase: the cobalt type NHase; Fe-NHase: the iron type NHase; gst-P14K: a gst tag fused at the N-terminus of P14K; his-P: the gene of the protein his-P14K; his-P14K: a his tag fused at the N-terminus of P14K; NHase: nitrile hydratase; P14K-his: a his tag fused th the C-terminus of P14K; P-his: the gene of the protein P14K-his.}

\section{Competing interests}

The authors declare that they have no competing interests.

\section{Authors' contributions}

$Y L$ and ZMZ designed the experiment including the NHase cloning, expression, point mutation and bioinformatics' calculation. WFS, LBZ and PC performed the experiment including the specific gene sequence analysis, cloning and expression, purification and bioinformatics' calculation. WFS and YL wrote and modified this manuscript. LLY, WWD and XGC cooperated during the experiments including providing instruments, strains, plasmids and bioinformatics' analysis. All authors read and approved this submission.

\section{Acknowledgements}

This work was supported by the Spring Sunshine Plan of the Ministry of Education of China (Z2015121), the National Natural Science Foundation of China (21506172), the Key Project of Xihua University of China (z1420524) and (z1420525), the Department of Education of Sichuan Province of China (15205445), the Key Laboratory program of Food and Biotechnology of Xihua University of China (szij2015-013) and (szjj2015-014).

\section{Author details}

${ }^{1}$ Key Laboratory of Food and Biotechnology, School of Food and Biotechnology, Xihua University, Chengdu 610039, China. ${ }^{2}$ School of Biochemical Engineering, Anhui Polytechnic University, Anhui 241000, China. ${ }^{3}$ Key Laboratory of Industrial Biotechnology, School of Biotechnology, Jiangnan University, Wuxi 214122, China.

Received: 23 August 2015 Accepted: 15 February 2016

Published online: 20 February 2016

\section{References}

1. Noguchi T, Nojiri M, Takei K, Odaka M, Kamiya N. Protonation structures of Cys-sulfinic and Cys-sulfenic acids in the photosensitive nitrile hydratase revealed by Fourier transform infrared spectroscopy. Biochemistry. 2003; 42(40):11642-50.

2. Kobayashi M, Shimizu S. Cobalt proteins. Eur J Biochem. 1999:261(1):1-9.

3. Liu Y, Cui W, Liu Z, Cui Y, Xia Y, Kobayashi M, et al. Effect of flexibility and positive charge of the C-terminal domain on the activator P14K function for nitrile hydratase in Pseudomonas putida. FEMS Microbiol Lett. 2014;352(1): 38-44.

4. Cameron RA, Sayed M, Cowan DA. Molecular analysis of the nitrile catabolism operon of the thermophile Bacillus pallidus RAPc8. Bba-Gen Subj. 2005;1725(1):35-46.

5. Liu Y, Cui W, Xia Y, Cui Y, Kobayashi M, Zhou Z. Self-subunit swapping occurs in another gene type of cobalt nitrile hydratase. PLoS One. 2012; 7(11):e50829. 
6. Zhou Z, Hashimoto Y, Shiraki K, Kobayashi M. Discovery of posttranslational maturation by self-subunit swapping. Proc Natl Acad Sci U S A. 2008; 105(39):14849-54.

7. Zhou ZM, Hashimoto Y, Cui TW, Washizawa Y, Mino H, Kobayashi M. Unique biogenesis of high-molecular mass multimeric metalloenzyme nitrile hydratase: intermediates and a proposed mechanism for self-subunit swapping maturation. Biochemistry. 2010;49(44):9638-48.

8. Zhou ZM, Hashimoto Y, Kobayashi M. Self-subunit swapping chaperone needed for the maturation of multimeric metalloenzyme nitrile hydratase by a subunit exchange mechanism also carries out the oxidation of the metal ligand cysteine residues and insertion of cobalt. J Biol Chem. 2009; 284(22):14930-8

9. Okamoto S, Van Petegem F, Patrauchan MA, Eltis LD. AnhE, a metallochaperone involved in the maturation of a cobalt-dependent nitrile hydratase. J Biol Chem. 2010:285(33):25126-33.

10. Wu S, Fallon RD, Payne MS. Over-production of stereoselective nitrile hydratase from Pseudomonas putida 5B in Escherichia coli: activity requires a novel downstream protein. Appl Microbiol Biotechnol. 1997;48(6):704-8.

11. Nishiyama M, Horinouchi S, Kobayashi M, Nagasawa T, Yamada H, Beppu T. Cloning and characterization of genes responsible for metabolism of nitrile compounds from Pseudomonas chlororaphis B23. J Bacteriol. 1991;173(8):2465-72.

12. Hashimoto $Y$, Nishiyama M, Horinouchi S, Beppu T. Nitrile hydratase gene from Rhodococcus sp. N-774 requirement for its downstream region for efficient expression. Biosci Biotechnol Biochem. 1994;58(10):1859-65.

13. Petrillo KL, Wu S, Hann EC, Cooling FB, Ben-Bassat A, Gavagan JE, et al. Over-expression in Escherichia coli of a thermally stable and regio-selective nitrile hydratase from Comamonas testosteroni 5-MGAM-4D. Appl Microbio Biotechnol. 2005;67(5):664-70.

14. Nagashima S, Nakasako M, Dohmae N, Tsujimura M, Takio K, Odaka M, et al. Novel non-heme iron center of nitrile hydratase with a claw setting of oxygen atoms. Nat Struct Mol Biol. 1998;5(5):347-51.

15. Murakami T, Nojiri M, Nakayama H, Dohmae N, Takio K, Odaka M, et al. Posttranslational modification is essential for catalytic activity of nitrile hydratase. Protein Sci. 2000;9(5):1024-30.

16. Yamanaka Y, Hashimoto K, Ohtaki A, Noguchi K, Yohda M, Odaka M. Kinetic and structural studies on roles of the serine ligand and a strictly conserved tyrosine residue in nitrile hydratase. J Biol Inorg Chem. 2010;15(5):655-65.

17. Yu H, Liu J, Shen Z. Modeling catalytic mechanism of nitrile hydratase by semi-empirical quantum mechanical calculation. J Mol Graph Model. 2008; 27(4):522-8

18. Mitra S, Holz RC. Unraveling the catalytic mechanism of nitrile hydratases. J Biol Chem. 2007;282(10):7397-404.

19. Liu Y, Cui W, Liu Z, Cui Y, Xia Y, Kobayashi M, et al. Enhancement of thermostability and product tolerance of Pseudomonas putida nitrile hydratase by fusing with self-assembling peptide. J Biosci Bioeng. 2014;118(3):249-52.

20. Cui Y, Cui W, Liu Z, Zhou L, Kobayashi M, Zhou Z. Improvement of stability of nitrile hydratase via protein fragment swapping. Biochem Bioph Res Co. 2014;450(1):401-8

21. Liu Y, Cui W, Fang Y, Yu Y, Cui Y, Xia Y, et al. Strategy for successful expression of the Pseudomonas putida nitrile hydratase activator P14K in Escherichia coli. BMC Biotechnol. 2013;13(1):48-54.

22. Nojiri M, Yohda M, Odaka M, Matsushita Y, Tsujimura M, Yoshida T, et al. Functional expression of nitrile hydratase in Escherichia coli: requirement of a nitrile hydratase activator and post-translational modification of a ligand cysteine. J Biochem. 1999;125(4):696-704.

23. Dougan $\mathrm{D}$, Truscott $\mathrm{K}$, Zeth $\mathrm{K}$. The bacterial $\mathrm{N}$-end rule pathway: expect the unexpected. Mol Microbiol. 2010;76(3):545-58.

24. Varshavsky A. The N-end rule pathway of protein degradation. Genes Cells 1997;2(1):13-28

25. Tobias JW, Shrader TE, Rocap G, Varshavsky A. The N-end rule in bacteria. Science. 1991;254(5036):1374-7.

26. Yokoyama S. Protein expression systems for structural genomics and proteomics. Curr Opin Chem Biol. 2003;7(1):39-43.

27. Schochetman G, Ou CY, Jones WK. Polymerase chain reaction. J Infect Dis. 1988;158(6):1154-1157.

28. Eswar N, Webb B, Marti-Renom MA, Madhusudhan M, Eramian D, Shen M et al. Comparative protein structure modeling using Modeller. Curr Protoc Protein Sci. 2006:Chapter 5:Unit 5.6. 5.6.1-5.6.30.

29. Damborský J, Prokop M, Koča J. TRITON: graphic software for rational engineering of enzymes. Trends Biochem Sci. 2001;26(1):71-3.

\section{Submit your next manuscript to BioMed Central and we will help you at every step:}

- We accept pre-submission inquiries

- Our selector tool helps you to find the most relevant journal

- We provide round the clock customer support

- Convenient online submission

- Thorough peer review

- Inclusion in PubMed and all major indexing services

- Maximum visibility for your research

Submit your manuscript at www.biomedcentral.com/submit 